\section{A Technique for Obtaining Undistorted Specimens of the Central Nervous System}

\section{K. M. LAURENCE* AND DEREK MARTIN}

From the Departments of Morbid Anatomy and of Medical Illustration, The Hospital for Sick Children, Great Ormond Street, London

\section{(RECEIVED FOR PUBlication AUGUST 20, 1958)}

The conventional methods used for the removal of the central nervous system may lead to distortion of the brain, and it is difficult to obtain an intact specimen in certain pathological conditions without employing some special technique. This is particularly true in cases of hydrocephalus where the "cerebral cortex" is frequently so thin that the brain collapses as soon as the skull cap is removed.

A special technique for the removal of the brain and spinal cord in situ within the bony cage was described by Martin (1952). There was one serious disadvantage in this method, as the cranial cavity had to be opened, with the possibility of distortion of its contents. The present method obviates such a possibility and is an improvement on that originally described.

\section{Method}

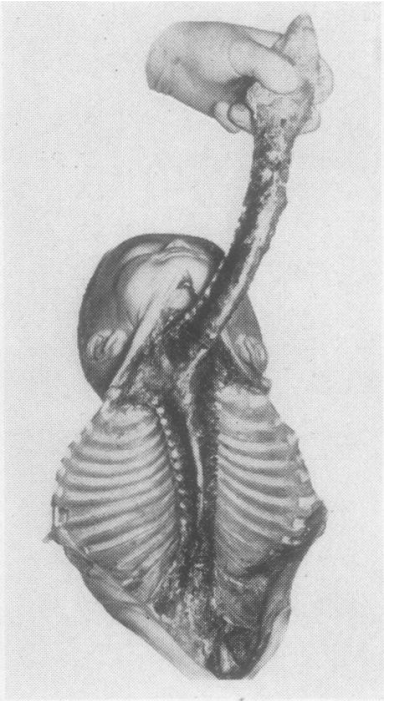

Fig. 1.-After disarticulation, the spinal column is dissected from the attached structures as far as the base of the skull.

*Present address: Department of Child Health, Llandough Hospital, Penarth, Glam.
Removal of Specimen.-The viscera are removed in the customary way to expose the entire length of the vertebral column. The sacro-iliac joints are disarticulated with a $\mathbf{s h}$ a r p cartilage knife or saw, and the costo-vertebral ligaments are cut. The vertebral column is then dissected away from skin and other structures as far as the base of the skull (Fig. 1). If a spina bifida cystica is present it is advisable to make incisions from behind through the skin around the lesion before the above procedures and remove the latter together with the vertebral column.

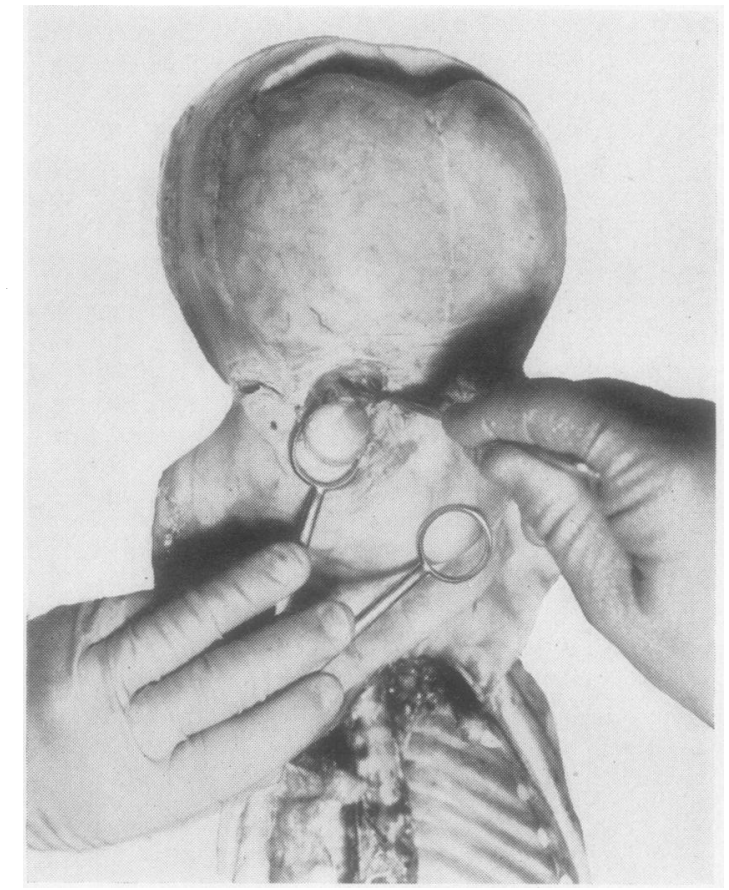

FIG. 2.-This shows the mobilization of the eye from the orbit.

With the body in the prone position, taking care to protect the facies from pressure, an incision is made from the vertex (anterior fontanelle) to the nape of the neck. The scalp is reflected from the calvarium as far as the ramus of the mandible, the attachments of the pinnae are severed, and the temporo-mandibular joints disarticulated.

The body is then placed in the supine position and the scalp is retracted over the face as far as the superior orbital margins and the bridge of the nose. Care must be taken to avoid cuts in the skin because of difficulties with subsequent reconstruction. The skin is then dissected from the bridge and sides of the nose, and the orbital septae are cut to expose the peri-orbital fat. The round handle of a pair of scissors is inserted behind the eyeball, which is retracted forward, and its muscles and nerves are severed (Fig. 2). The eyeball thus remains attached to the skin by its conjunctiva and subconjunctival tissues only. The scalp and attached eyes are deflected forwards and an oblique saw-cut is made across the bridge of the nose and zygoma to the pharynx (Fig. 3). The skull and the vertebral column are finally freed from the rest of the body by cutting the remaining fascial strands and muscles. 

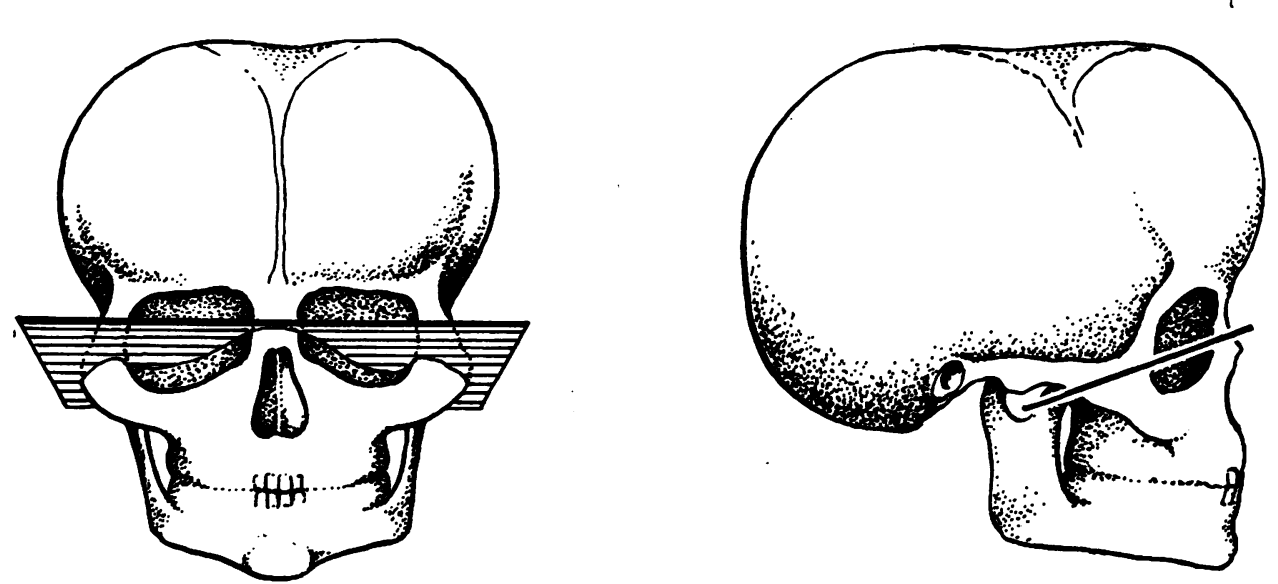

FIG. 3.-Diagrams to illustrate the plane of the saw cut through the facial bones.

Altogether these procedures should not take longer than half an hour in a child, although in an adult more time may be required.

Fixation.-Fixation is critical and necessitates some care. The best results are obtained when the carotid arteries are perfused with saline solution and then $10 \%$ formol saline before necropsy. Unfortunately, this technique may produce wrinkling and shrinkage of the facial skin, which makes the subsequent reconstruction difficult. This method, therefore, is only applicable to selected cases.

More generally, fixation is achieved after removal of the specimen by needling the ventricular system either through the anterior fontanelle or a burr hole and replacing the bulk of the cerebrospinal fluid with $15 \%$ formol saline. The needle is then partly withdrawn and a small quantity of the fixative is instilled in the subdural space. Fixative is also introduced into the lumbar theca in much the same way as a lumbar puncture is performed. The whole specimen is then placed in a bath of $10 \%$ formol saline in, as nearly as possible, its correct anatomical position, and fixation is allowed for at least one week, but preferably for three.

Reconstruction.-The reconstruction of the body is carried out in the usual way. The vertebral column is replaced by a measured wooden prop, and a representation of the skull is modelled in moist lint and cotton wool. After suitable packing, the abdomen and neck incisions are sewn up. The head is best fashioned into the correct shape upon the measured prop before placing the latter in position, using the specimen as a guide to the exact shape required. Two depressions are made for the orbits, into which the eyes can be inserted. The scalp is then partially stitched up and moulding is completed with pledgets of moist cotton wool. Head measurements made before the necropsy may be of assistance. With the above technique, especially in cases of hydrocephalus, subsequent appearances may indeed represent a cosmetic improvement on the original.
Dissection of the Specimen.-The precise method adopted for the dissection is obviously largely dictated by the suspected lesion and the structures to be studied primarily. In practice, the most satisfactory method is to make a mid-line saw-cut through the skull, spines, and vertebral bodies, without damaging the dura. The hemisection of the vertebrae, starting at the coccyx, is completed with a cartilage knife.

After severing the spinal nerve roots as closely as possible to their entry into the intervertebral foraminae, one half of the column is removed, leaving the dura intact. One half of the calvarium is then removed piecemeal with bone forceps and saw, without damaging the underlying dura (Fig. 4). The latter is then stripped off the exposed portion of the cord and brain ; the tentorium, transverse sinus, falx cerebri, and the superior sagittal sinus are left in situ. The exposed surfaces of the brain and cord can then be examined in detail. Further dissections are made, or the brain and cord removed in toto, depending on the nature of the findings. For general purposes of demonstration, the method of choice is a mid-sagittal section achieved by carefully separating the cerebral hemispheres and then cutting down the cerebrospinal axis (Fig. 5).

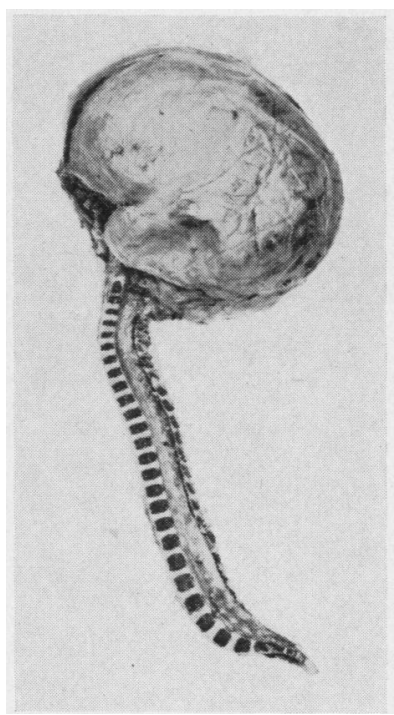

FIG. 4.-The specimen after removal of half the bony cage; the dura remains intact. 


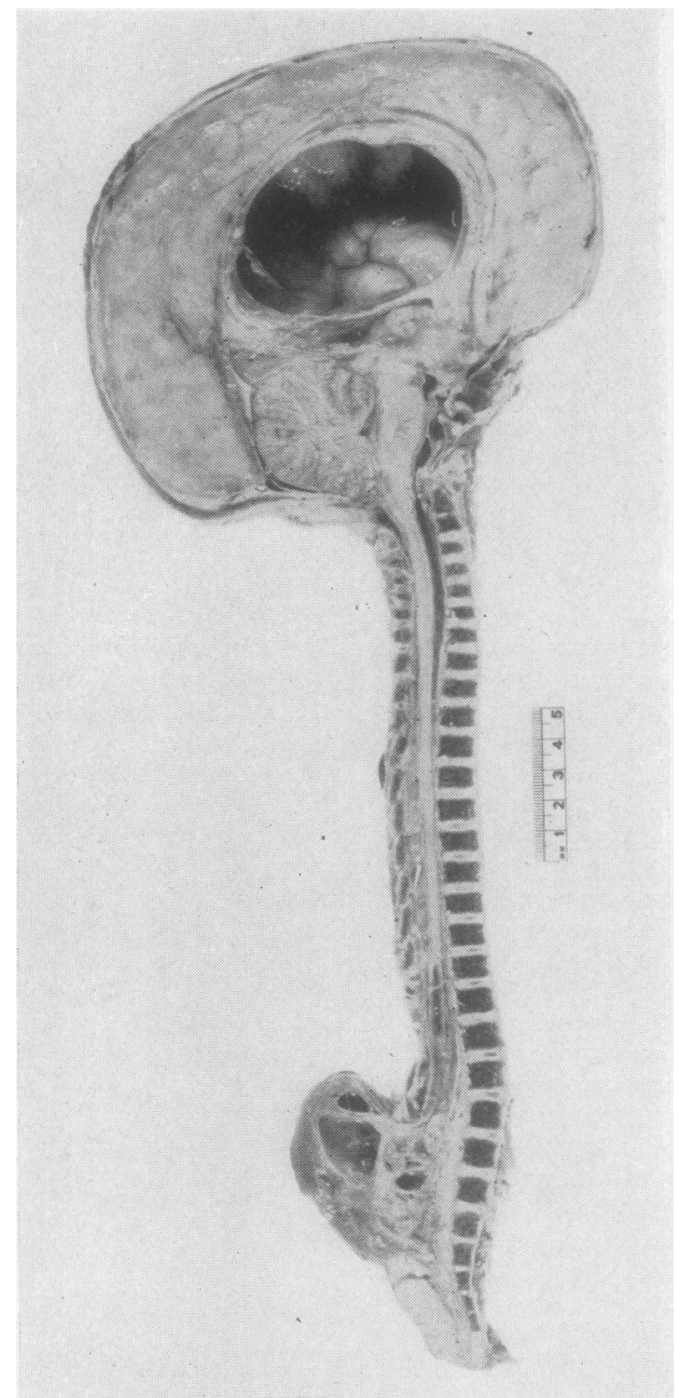

Fig. 5-A complete hemisection of the cential nervous system showing hydrocephalus, small Arnold-Chiari malformation, and a sa:ral myelocele.

\section{Discussion}

This method permits the preservation of precise anatomical relationships, such as those of the basal cisterns, without the possibility of distortion. It is also ideal for obtaining specimens for demonstration and research. If an immediate opinion on the central nervous system is required, the technique is obviously not practical, nor is it recommended for routine use. This method is, however, ideal for the investigation of such problems as hydrocephalus. A brain with attenuated cerebral tissue may be examined, when fixed, without danger of distortion after removal from the cranium. This is probably the only way whereby the basal cisterns may be studied post mortem. Minor degrees of tentorial herniation, otherwise often missed (Laurence, 1957), can also be easily recognized. The Arnold-Chiari malformation is preserved in its correct relationship to the surrounding bony structures. and even minor deformities can be demonstrated. The study of other malformations, including cranial spina bifida, agenesis of the corpus callosum, and porencephaly, and also cerebral tumours is greatly facilitated. By this method the brain can also be studied after recent extensive intracranial operations. which may make examination by conventional procedures difficult.

\section{Summary}

A method of removing the central nervous system within the bony cage is described. This enables undistorted specimens to be examined in their correct anatomical relationships, and is an ideal technique for teaching and research. Some special applications of the method are suggested.

We wish to thank Dr. M. Bodian for his advice and permission to publish and Mr. D. Lyth for Fig. 3 : One of us (K. M. L.) is indebted to the Research Committee of The Hospital for Sick Children, Great Ormond Street, for support.

\section{REFERENCES}

Laurence, K. M. (1957). The Cerebrospinal Fluid, p. 280. Ed. G. E. W. Wolstenholme and C. M. O'Connor. Churchill, London.

Martin, D. (1952). Med. biol. Ill., 2, 260. 